barrier that is essential for many cellular functions ${ }^{1}$, and it contains proteins that are crucial to controlling the uptake of nutrients, the release of waste and the production of energy in the form of molecules of ATP. By distorting the membrane, the retinoid molecules probably affect the import and export of solutes, as well as several other essential cellular functions that rely on membrane integrity. However, a mode of antibacterial action that involves simply attacking the bacterial membrane is not guaranteed to kill persister cells. For example, the authors report that the membrane-targeting antibiotics nigericin and valinomycin do not kill MRSA persister cells.

Through computer simulations, the authors explored how the retinoid molecules might interact with the bacterial membrane. They determined that the polar side groups of CD437 and CD1530 could bind to the hydrophilic heads of phospholipids in the membrane, enabling the retinoid molecules to lodge in the lipid bilayer of a bacterium. Such simulations are a powerful tool that could be used to guide the optimization of antibiotics that can selectively attack the lipid bilayer of bacterial membranes without disrupting their mammalian counterparts and causing toxicity to patients.

A major concern is how to optimize small molecules such as the retinoids to enable such selectivity. Although the authors found that CD437 and CD1530 did not destroy the lipid membranes of human red blood cells, the

molecules were able to kill human liver-cancer cells grown in vitro, which is consistent with the previously reported anticancer properties of the retinoids ${ }^{6}$.

The authors generated structural variants of CD437, producing a compound they called analogue 2 that did not kill normal or cancerous human liver cells grown in vitro, but did retain the ability to kill MRSA persister cells (Fig. 1). In experiments in mice, analogue 2 remained in circulation in the animals' bodies for several hours at

"Some bacterial cells enter a metabolically inactive or dormant state to become persister cells." high enough concentrations to kill MRSA persister cells, but did not give rise to signs of toxicity such as liver or kidney damage. Remarkably, the authors showed in mice that analogue 2 could tackle what would generally be considered to be a treatment-resistant form of MRSA. This animal model mimics chronic infection with MRSA in immunocompromised people, for whom the prognosis is poor with conventional antibiotic treatments such as vancomycin because of the large number of MRSA persister cells that are present ${ }^{4,7}$.

The authors found that the effects of analogue 2 on bacterial infections could be boosted by the presence of the antibiotic gentamicin, an inhibitor of bacterial protein synthesis that lacks activity against persister cells. It will be interesting to determine whether
MRSA persister cells respond to the retinoids by reactivating their cellular metabolism, thereby making them more susceptible to being killed by drugs such as gentamicin that would otherwise be ineffective.

Molecules such as analogue 2 might be suitable candidates for drugs that decrease the time required to successfully treat chronic infections that are characterized by high loads of dormant bacteria. In an era in which the development of antibiotics is struggling to keep pace with the spread of resistant bacteria, the identification of compounds such as analogue 2 could help researchers to win victories in the long fight against bacterial infectious diseases.

Julian G. Hurdle and Aditi Deshpande are in the Center for Infectious and Inflammatory Diseases, Institute of Biosciences and Technology, Texas A\&M Health Science Center, Houston, Texas 77030, USA. e-mails: jhurdle@ibt.tamhsc.edu; adeshpande@medicine.tamhsc.edu

1. Hurdle, J. G., O'Neill, A. J., Chopra, I. \& Lee, R. E. Nature Rev. Microbiol. 9, 62-75 (2011).

2. Kim, W. et al. Nature 556, 103-107 (2018)

3. Costerton, J. W., Stewart, P. S. \& Greenberg, E. P. Science 284, 1318-1322 (1999).

4. Lewis, K. Nature Rev. Microbiol. 5, 48-56 (2007).

5. Dantes, R. et al. JAMA Int. Med. 173, 1970-1978 (2013).

6. Álvarez, R., Vaz, B., Gronemeyer, H. \& de Lera, Á. R. Chem. Rev. 114, 1-125 (2014)

7. Conlon, B. P. et al. Nature 503, 365-370 (2013).

This article was published online on 28 March 2018.

\title{
Observations of the birth of crystals
}

\section{Different forms of molecular crystals often have distinct properties, which can greatly influence their potential applications. A way of controlling the crystal form of a protein has now been reported. SEE LETTER P.89}

\section{ROBERT G. ALBERSTEIN \& F. AKIF TEZCAN}

$\mathrm{T}$ he second law of thermodynamics dictates that all things tend towards disorder. Yet molecules and other microscopic particles in liquids frequently arrange themselves into perfectly ordered arrays - crystals - without violating this law. Moreover, a given molecule can often arrange itself into more than one type of array, producing different crystal forms known as polymorphs. These polymorphs can have remarkably different properties despite being composed of the same building blocks.
On page 89 , Van Driessche et al. ${ }^{1}$ report experimental observations of protein molecules as they begin to assemble into clusters that then evolve into distinct polymorphs. Their findings bring fresh insight to the important processes of crystal formation and growth, and polymorph selection.

The everyday consequences of crystal polymorphism are perhaps highlighted best by pharmaceutical drugs, most of which are administered as crystalline solids ${ }^{2}$. Polymorphs of drug molecules often exhibit considerable variation in their ease of manufacture, their shelf life and - crucially — their physical and chemical properties, which greatly influence their physiological efficacies ${ }^{2}$. The selection and manufacture of appropriate polymorphs is a major and costly component of the drug-development process, yet processes for polymorph selection are largely conducted on the basis of trial and error, rather than through molecular design.

The development of rational approaches for the design and control of crystal growth, as well as for polymorph selection, requires an understanding of nucleation - the initial stages of crystallization, in which the building blocks begin to form clusters known as nuclei. Unfortunately, there are two main hurdles to capturing and characterizing crystals at birth. First, the nuclei are typically too small to be visualized in $3 \mathrm{D}$ space using most experimental methods, especially when they consist of atoms or small molecules. Second, such nuclei are, by definition, unstable and therefore form only transiently.

To address the first issue, Van Driessche and colleagues used the protein glucose isomerase (GI) as a building block, the box-like shape and nanometre dimensions of which make it relatively easy to identify using a technique called cryo-transmission electron microscopy (cryoTEM). And to overcome the second issue, they 


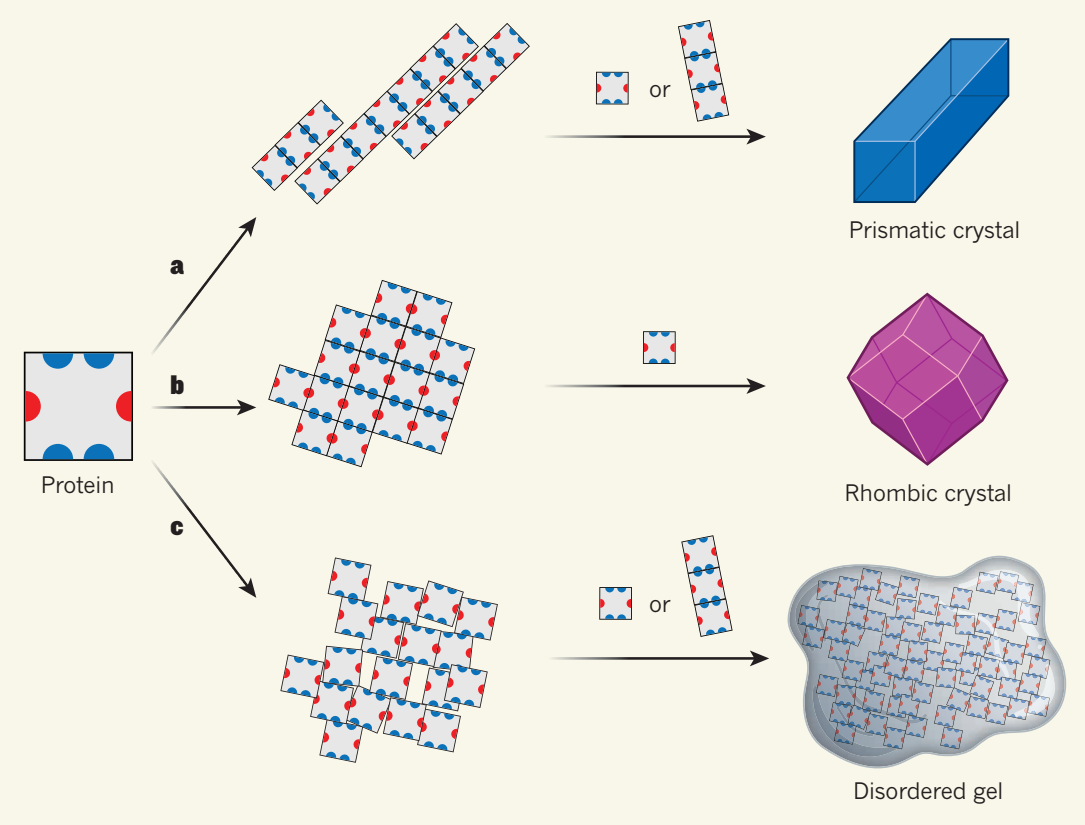

Figure 1 | Nucleation determines crystal growth and structure. Van Driessche et al. ${ }^{1}$ have worked out how interactions between molecules of a box-shaped protein affect its nucleation (the formation of molecular clusters known as nuclei that act as 'seeds' for crystals) and, in turn, the growth and structure of crystals of the protein. Coloured semicircles represent sites of interaction on the molecules. a, Under conditions in which interactions between blue sites are stronger than those between red sites, rod-shaped nuclei form. Subsequent crystal growth occurs through the addition of either individual proteins or rods, and results in the formation of prismatic crystals. $\mathbf{b}$, When the blue interactions are as strong as the red interactions, the nuclei have smaller aspect ratios than in a and grow equally in all directions through the addition of individual proteins. The resulting crystals are rhombic. c, Strong but indiscriminate interactions cause the molecules to form disordered clusters, which produce similarly disordered gels. (Adapted from a figure supplied by R. G. Alberstein.)

used a protocol in which protein samples were rapidly frozen to about $-183^{\circ} \mathrm{C}$, a temperature at which essentially all motion by GI molecules stops. This enabled them to take snapshots of the crystallization process at time intervals ranging from seconds to minutes. The authors thus captured the nucleation and growth of GI crystals with sufficiently high temporal and spatial resolution for them work out how the emergent crystal morphologies depend on specific interactions formed between GI molecules.

To initiate crystallization, the authors mixed GI with ammonium sulfate or polyethylene glycol (PEG), which are commonly used as agents for modulating protein solubility and the strength of interactions between proteins. They observed that, at high concentrations of ammonium sulfate, GI molecules rapidly line up into rods that are a few proteins in length. These rods then align side by side, while also growing longer, to yield macroscopic crystals with a rectangular, prism-like shape that resembles the rod-shaped nuclei (Fig. 1a). By contrast, low concentrations of PEG cause the GI nuclei to grow more slowly and evenly in all dimensions, to yield rhombic crystals with a diamond-like shape (Fig. 1b). And at high concentrations of PEG, the GI molecules become locked into structures that are best described as disordered gels rather than crystals (Fig. 1c).
Van Driessche and colleagues' cryo-TEM images of the GI nuclei are detailed enough to be compared with known 3D atomic structures of GI crystals. Such comparisons enabled the authors to propose plausible models for the arrangement of GI molecules in the nuclei, as well as for the specific interactions between the molecules. The authors then designed mutants of GI in which a key amino-acid residue at each interaction site in the various nuclei was replaced with another residue. The mutant proteins were unable to form their corresponding polymorph and either produced the alternative crystal polymorph or aggregated into gels, depending on the conditions. These observations validated the proposed structural models and demonstrated that polymorphs could be selected predictably.

Classical nucleation theory (CNT) posits that crystallization must start with the formation of a nucleus that has the same molecular order and arrangement as do the macroscopic crystals, and that the building blocks are added one by one to the nucleus ${ }^{3}$. In the past two to three decades, CNT has been largely superseded by two-step or multistep nucleation models in which an amorphous, high-density liquid phase forms, and then transitions into either a single crystalline domain (as occurs in some inorganic nucleation processes ${ }^{4}$ ) or numerous small, locally ordered clusters, which align to form growing crystals by a process known as oriented attachment. In light of these competing views, characterization of the nucleation events for various systems has been the subject of much experimental and theoretical work ${ }^{3}$.

Van Driessche and co-workers' observations, particularly of the prismatic GI crystals, indicate that elements of both CNT and multistep nucleation might be at play. The authors uncover no evidence of an amorphous liquid phase, and the smallest GI rods, which they captured at very early time points (just 20 seconds after the addition of ammonium sulfate), have the same crystalline registry as the mature crystals - findings that are in accord with CNT. However, the rods undergo oriented attachment during crystal maturation, as in the multistep model. The picture is less clear for the rhombic GI crystals, the precursors of which become observable only after several minutes - at which point they are already bigger than a nucleus. It could be that an amorphous, high-density liquid phase does form in this case but escapes detection because of its instability or the low contrast of the cryo-TEM images.

We can, however, be certain that rapidly advancing techniques such as cryo-TEM, liquid-cell transmission electron microscopy $^{5,6}$ and in situ atomic force microscopy ${ }^{7}$, complemented by theory and computational modelling ${ }^{8}$, will continue to provide intriguing results with which to refine our understanding of crystal nucleation and growth. More practically, Van Driessche and colleagues' study shows that the crystallization or self-assembly pathways of proteins can be rationally engineered at the molecular level to obtain a desired polymorph. This feat is particularly notable, given that the number of protein-based agents being used as pharmaceutical drugs is increasing, and that synthetic protein assemblies and crystals are being designed and constructed to have unusual and potentially useful properties ${ }^{10}$.

Robert G. Alberstein and F. Akif Tezcan are in the Department of Chemistry and Biochemistry, University of California, San Diego, La Jolla, California 92093, USA. e-mail:tezcan@ucsd.edu

1. Van Driessche, A. E. S. et al. Nature 556, 89-94 (2018).

2. Lee, A. Y., Erdemir, D. \& Myerson, A. S. Annu. Rev. Chem. Biomol. Eng. 2, 259-280 (2011).

3. De Yoreo, J. J. et al. Science 349, aaa6760 (2015).

4. Pouget, E. M. et al. Science 323, 1455-1458 (2009).

5. Yamazaki, Y. et al. Proc. Natl Acad. Sci. USA 114 2154-2159 (2017).

6. Parent, L. R. et al. J. Am. Chem. Soc. 139, 17140-17151 (2017).

7. Chung, S., Shin, S.-H., Bertozzi, C. R. \& De Yoreo, J. J. Proc. Natl Acad. Sci. USA 107, 16536-16541 (2010)

8. Whitelam, S. Phys. Rev. Lett. 105, 088102 (2010).

9. Walsh, G. Nature Biotechnol. 28, 917-924 (2010). 10.Suzuki, Y. et al. Nature 533, 369-373 (2016). 